\title{
Crystal structure of 11-methyl-7,11-diazatricyclo[7.3.1.0 $\left.{ }^{2,7}\right]$ tri- decan-13-ols, $\mathrm{C}_{12} \mathrm{H}_{22} \mathrm{~N}_{2} \mathrm{O}$, solid state conformation of syn- and trans-isomers
}

\author{
H. J. Lindner ${ }^{1}$, S. Foro ${ }^{* .1}$, P. Nemes ${ }^{\text {II }}$ and P. Scheiber ${ }^{\mathrm{II}}$ \\ 1 Technische Universität Darmstadt, Institut für Organische Chemie, Petersenstr. 22, D-64287 Darmstadt, Germany \\ "University of Veterinary Science, Department of Chemistry, Budapest, Hungary
}

Received December 15, 1999, accepted in final form August 17, 2001, CCDC-No. 133810 and CCDC-No. 133811

\author{
Abstract \\ $\mathrm{C}_{12} \mathrm{H}_{22} \mathrm{~N}_{2} \mathrm{O}$, monoclinic, $C 12 / c 1$ (No. 15), $a=21.125(3) \AA$ \\ $b=14.102(2) \AA, c=20.172(4) \AA, \beta=126.45(1)^{\circ}$, \\ $V=4833.9 \AA^{3}, Z=16, R_{\mathrm{gt}}(F)=0.054, w R_{\mathrm{obs}}\left(F^{2}\right)=0.142$, \\ $T=298 \mathrm{~K}$. \\ $\mathrm{C}_{12} \mathrm{H}_{22} \mathrm{~N}_{2} \mathrm{O}$, monoclinic, $P 12{ }_{1} / c 1$ (No. 14), $a=12.527(3) \AA$, \\ $b=11.171(5) \AA, c=17.757(4) \AA, \beta=106.20(2)^{\circ}$, \\ $V=2386.3 \AA^{3}, Z=8, R_{\mathrm{gt}}(\mathrm{F})=0.053, w R_{\mathrm{obs}}\left(F^{2}\right)=0.118$, \\ $T=300 \mathrm{~K}$.
}

\section{Source of material}

The substances were prepared as described previously [1]. Colorless prisms of anti-11-methyl-7,11-diazetricyclo [3.3.1.0 2,7 ]tridecan-13-ol ( $\alpha$-isomer) were grown by slow recrystallisation from butanone solution at ambient temperature ( $\mathrm{mp} 432 \mathrm{~K}$ ). Colorless needles of $s y n-11$-methyl-7,11-diazatricyclo [7.3.1.0 $\left.0^{2,7}\right]$ tridecan-13-ol ( $\beta$-isomer) were obtained from acetone (mp $418 \mathrm{~K}$ ).

\section{Experimental details}

In the $\alpha$-isomer all hydrogen atoms were located in difference Fourier maps and refined isotropically, in the $\beta$-isomer the hydrogen atoms of the hydroxy groups were found in a difference fourier map and refined isotropically, the other hydrogen atoms were positioned geometrically.

\section{Discussion}

Owing to the easy chair-boat interconversion of the sixmembered saturated rings and the repulsion between their endo groups/atoms at the 3 and 7 positions the conformation of the bicyclo [3.3.1]nonanes has attracted considerable interest. It seems reasonable to expect the so-called chair-chair conformation to be preferred to either the boat-chair or boat-boat conformation. While the carbocyclic compound [2] and the 3-aza analogue [3] adopt a chair-chair conformation, among the 3,7-dihetera (N, S, Se) analogues [4-7] also chair-boat forms can occur in crystalline state reflecting the strength of the transannular $3 / 7$ repulsion. Due to the effects on the cardiovascular system [8] the 3,7-diazabicyclo[3.3.1]nonanes were particularly thoroughly studied. Their bicyclic unit constitutes the B and $C$ rings of the molecules of the tri- and tetracyclic lupine alkaloids cytisine and sparteine, respectively. The saturated diazatricyclo[7.3.1.0 $\left.0^{2,7}\right]$ tridecanes and some other congeners were also

synthesized [1] and characterized [9] to assume an all chair or chair-chair-boat conformation in solution depending on the nature and steric position of the $\mathrm{C}(13)$ substituent and/or the solvent used. Particularly, the epimeric C(13) title compounds have been found to display striking differences in chloroform solution [9]. While cytisine itself including an $\alpha$-pyridone moiety has been subjected to structure elucidation by $\mathrm{X}$-ray diffraction in three independent works [10-12], all showing ring $C$ with chair conformation its saturated derivatives like the title compounds were not investigated yet.

\section{1. anti-11-Methyl-7,11-diazetricyclo [3.3.1.0 $\left.{ }^{2,7}\right]$ tridecan-13-ol}

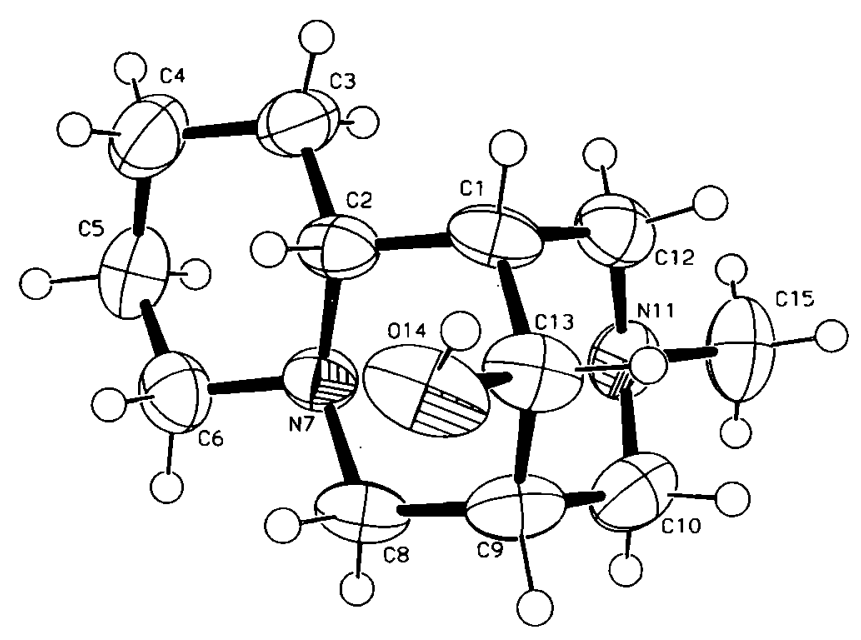

Table 1. Data collection and handling.

Crystal:

Wavelength:

$\mu$ :

Diffractometer, scan mode:

$2 \theta_{\max }$ :

$N(h k l)_{\text {measured, }} N(h k l)_{\text {unique: }}$

Criterion for $I_{\text {obs, }} N(h k l)_{\text {gl }}$ :

$N($ param) refined:

Programs: colourless prism,

size $0.55 \times 0.325 \times 0.325 \mathrm{~mm}$

Mo $K_{\alpha}$ radiation $(0.71093 \AA$ )

$0.7 \mathrm{~cm}^{-1}$

Nonuis CAD4, $\omega / 2 \theta$

$45.94^{\circ}$

3465,3351

$I_{\mathrm{obs}}>2 \sigma\left(I_{\mathrm{obs}}\right), 2608$

447

SHELXS-86 [13], SHELXL-93 [14], PLUTON93 [15], ORTEPIII [16]

* Correspondence author

(e-mail: sabine@friedel.st.mw.tu-darmstadt.de) 
Table 2. Atomic coordinates and displacement parameters (in $\AA^{2}$ ).

\begin{tabular}{llllll}
\hline Atom & Site & $x$ & $y$ & $z$ & $U_{\text {iso }}$ \\
\hline H(1) & $8 f$ & $0.263(1)$ & $-0.014(2)$ & $0.624(2)$ & $0.053(7)$ \\
H(2) & $8 f$ & $0.365(1)$ & $0.044(2)$ & $0.628(2)$ & $0.048(6)$ \\
H(31) & $8 f$ & $0.341(2)$ & $-0.155(2)$ & $0.581(2)$ & $0.056(7)$ \\
H(32) & $8 f$ & $0.376(2)$ & $-0.115(2)$ & $0.666(2)$ & $0.069(9)$ \\
H(41) & $8 f$ & $0.488(2)$ & $-0.053(2)$ & $0.678(2)$ & $0.078(9)$ \\
H(42) & $8 f$ & $0.478(2)$ & $-0.163(2)$ & $0.652(2)$ & $0.08(1)$ \\
H(51) & $8 f$ & $0.497(2)$ & $-0.069(2)$ & $0.569(2)$ & $0.09(1)$ \\
H(52) & $8 f$ & $0.412(2)$ & $-0.127(2)$ & $0.514(2)$ & $0.077(9)$ \\
H(61) & $8 f$ & $0.388(2)$ & $0.038(2)$ & $0.467(2)$ & $0.069(8)$ \\
H(62) & $8 f$ & $0.427(2)$ & $0.067(2)$ & $0.559(2)$ & $0.065(8)$ \\
H(81) & $8 f$ & $0.317(2)$ & $0.151(2)$ & $0.511(2)$ & $0.062(8)$ \\
H(82) & $8 f$ & $0.268(1)$ & $0.108(2)$ & $0.417(2)$ & $0.053(7)$ \\
H(9) & $8 f$ & $0.183(2)$ & $0.175(2)$ & $0.442(2)$ & $0.061(7)$ \\
H(101) & $8 f$ & $0.092(2)$ & $0.057(2)$ & $0.399(2)$ & $0.072(9)$ \\
H(102) & $8 f$ & $0.128(2)$ & $0.050(2)$ & $0.349(2)$ & $0.067(8)$ \\
H(121) & $8 f$ & $0.148(2)$ & $-0.072(2)$ & $0.521(2)$ & $0.057(7)$ \\
H(122) & $8 f$ & $0.207(2)$ & $-0.139(2)$ & $0.528(2)$ & $0.062(8)$ \\
H(13) & $8 f$ & $0.167(2)$ & $0.098(2)$ & $0.537(2)$ & $0.059(8)$ \\
H(14) & $8 f$ & $0.265(2)$ & $0.170(2)$ & $0.636(2)$ & $0.07(1)$ \\
H(151) & $8 f$ & $0.051(2)$ & $-0.102(2)$ & $0.386(2)$ & $0.09(1)$ \\
H(152) & $8 f$ & $0.077(2)$ & $-0.102(2)$ & $0.320(2)$ & $0.09(1)$ \\
H(153) & $8 f$ & $0.107(2)$ & $-0.186(3)$ & $0.391(3)$ & $0.12(2)$ \\
& & & & & \\
& & & & &
\end{tabular}

Table 2. Continued.

\begin{tabular}{llllll}
\hline Atom & Site & $x$ & $y$ & $z$ & $U_{\text {iso }}$ \\
\hline & & & & & \\
H(1A) & $8 f$ & $0.245(2)$ & $-0.327(2)$ & $0.321(2)$ & $0.060(7)$ \\
H(2A) & $8 f$ & $0.353(2)$ & $-0.240(2)$ & $0.372(2)$ & $0.065(8)$ \\
H(3A) & $8 f$ & $0.316(2)$ & $-0.336(2)$ & $0.233(3)$ & $0.09(1)$ \\
H(3B) & $8 f$ & $0.348(2)$ & $-0.387(3)$ & $0.316(2)$ & $0.09(1)$ \\
H(4A) & $8 f$ & $0.468(3)$ & $-0.309(3)$ & $0.377(3)$ & $0.13(1)$ \\
H(4B) & $8 f$ & $0.456(3)$ & $-0.352(4)$ & $0.302(3)$ & $0.16(2)$ \\
H(5A) & $8 f$ & $0.410(3)$ & $-0.224(3)$ & $0.229(3)$ & $0.13(2)$ \\
H(5B) & $8 f$ & $0.488(3)$ & $-0.190(3)$ & $0.307(3)$ & $0.14(2)$ \\
H(6A) & $8 f$ & $0.430(2)$ & $-0.123(3)$ & $0.362(3)$ & $0.10(1)$ \\
H(6B) & $8 f$ & $0.399(3)$ & $-0.076(3)$ & $0.283(3)$ & $0.13(2)$ \\
H(8A) & $8 f$ & $0.322(2)$ & $-0.065(2)$ & $0.348(2)$ & $0.066(8)$ \\
H(8B) & $8 f$ & $0.279(2)$ & $-0.025(2)$ & $0.255(2)$ & $0.064(8)$ \\
H(9A) & $8 f$ & $0.192(2)$ & $-0.046(2)$ & $0.290(2)$ & $0.067(8)$ \\
H(10A) & $8 f$ & $0.135(1)$ & $-0.068(2)$ & $0.146(2)$ & $0.054(7)$ \\
H(10B) & $8 f$ & $0.090(2)$ & $-0.124(3)$ & $0.175(2)$ & $0.10(1)$ \\
H(12A) & $8 f$ & $0.128(2)$ & $-0.313(2)$ & $0.198(2)$ & $0.069(8)$ \\
H(12B) & $8 f$ & $0.188(2)$ & $-0.341(2)$ & $0.179(2)$ & $0.065(8)$ \\
H(13A) & $8 f$ & $0.162(2)$ & $-0.204(2)$ & $0.307(2)$ & $0.061(8)$ \\
H(14A) & $8 f$ & $0.256(2)$ & $-0.133(2)$ & $0.418(2)$ & $0.07(1)$ \\
H(15A) & $8 f$ & $0.041(2)$ & $-0.242(2)$ & $0.070(2)$ & $0.073(9)$ \\
H(15B) & $8 f$ & $0.070(2)$ & $-0.163(2)$ & $0.037(2)$ & $0.071(9)$ \\
H(15C) & $8 f$ & $0.095(2)$ & $-0.282(3)$ & $0.041(3)$ & $0.12(2)$
\end{tabular}

Table 3. Atomic coordinates and displacement parameters (in $\AA^{2}$ ).

\begin{tabular}{|c|c|c|c|c|c|c|c|c|c|c|}
\hline Atom & Site & $x$ & $y$ & $z$ & $U_{11}$ & $U_{22}$ & $U_{33}$ & $U_{12}$ & $U_{13}$ & $U_{23}$ \\
\hline$C(1)$ & $8 f$ & $0.2547(2)$ & $-0.0022(2)$ & $0.5746(2)$ & $0.077(2)$ & $0.039(1)$ & $0.042(1)$ & $-0.000(1)$ & $0.044(1)$ & $0.001(1)$ \\
\hline$C(2)$ & $8 f$ & $0.3318(2)$ & $-0.0086(2)$ & $0.5858(1)$ & $0.057(2)$ & $0.040(1)$ & $0.034(1)$ & $-0.006(1)$ & $0.025(1)$ & $-0.002(1)$ \\
\hline$C(3)$ & $8 f$ & $0.3735(2)$ & $-0.1023(2)$ & $0.6198(2)$ & $0.058(2)$ & $0.056(2)$ & $0.044(2)$ & $0.001(1)$ & $0.021(1)$ & $0.009(1)$ \\
\hline$C(4)$ & $8 f$ & $0.4530(2)$ & $-0.1020(3)$ & $0.6348(2)$ & $0.050(2)$ & $0.068(2)$ & $0.069(2)$ & $0.006(2)$ & $0.020(2)$ & $0.009(2)$ \\
\hline$C(5)$ & $8 f$ & $0.4429(2)$ & $-0.0753(2)$ & $0.5569(2)$ & $0.045(2)$ & $0.080(2)$ & $0.071(2)$ & $0.000(2)$ & $0.029(2)$ & $-0.009(2)$ \\
\hline$C(6)$ & $8 f$ & $0.3975(2)$ & $0.0161(2)$ & $0.5224(2)$ & $0.054(2)$ & $0.061(2)$ & $0.053(2)$ & $-0.012(1)$ & $0.031(1)$ & $-0.010(1)$ \\
\hline$N(7)$ & $8 f$ & $0.3215(1)$ & $0.0118(1)$ & $0.5083(1)$ & $0.051(1)$ & $0.043(1)$ & $0.037(1)$ & $-0.0006(9)$ & $0.0272(9)$ & $-0.0004(8)$ \\
\hline$C(8)$ & $8 f$ & $0.2794(2)$ & $0.1014(2)$ & $0.4717(2)$ & $0.077(2)$ & $0.041(1)$ & $0.045(2)$ & $0.004(1)$ & $0.043(1)$ & $0.007(1)$ \\
\hline$C(9)$ & $8 f$ & $0.2044(2)$ & $0.1104(2)$ & $0.4634(2)$ & $0.077(2)$ & $0.041(1)$ & $0.049(2)$ & $0.016(1)$ & $0.039(1)$ & $0.010(1)$ \\
\hline$C(10)$ & $8 f$ & $0.1404(2)$ & $0.0410(2)$ & $0.4031(2)$ & $0.060(2)$ & $0.070(2)$ & $0.054(2)$ & $0.021(2)$ & $0.031(2)$ & $0.011(2)$ \\
\hline$N(11)$ & $8 f$ & $0.1606(1)$ & $-0.0578(2)$ & $0.4289(1)$ & $0.046(1)$ & $0.054(1)$ & $0.050(1)$ & $0.001(1)$ & $0.026(1)$ & $-0.006(1)$ \\
\hline$C(12)$ & $8 f$ & $0.1905(2)$ & $-0.0729(2)$ & $0.5147(2)$ & $0.061(2)$ & $0.052(2)$ & $0.058(2)$ & $0.003(1)$ & $0.042(1)$ & $0.003(1)$ \\
\hline$C(13)$ & $8 f$ & $0.2196(2)$ & $0.0970(2)$ & $0.5465(2)$ & $0.078(2)$ & $0.045(1)$ & $0.057(2)$ & $0.007(1)$ & $0.049(2)$ & $0.001(1)$ \\
\hline$O(14)$ & $8 f$ & $0.2704(1)$ & $0.1696(1)$ & $0.6012(1)$ & $0.117(2)$ & $0.045(1)$ & $0.065(1)$ & $-0.009(1)$ & $0.070(1)$ & $-0.0123(9)$ \\
\hline$C(15)$ & $8 f$ & $0.0925(2)$ & $-0.1185(3)$ & $0.3753(3)$ & $0.048(2)$ & $0.098(3)$ & $0.078(2)$ & $-0.014(2)$ & $0.033(2)$ & $-0.025(2)$ \\
\hline$C(1 A)$ & $8 f$ & $0.2388(2)$ & $-0.2687(2)$ & $0.2916(2)$ & $0.072(2)$ & $0.036(1)$ & $0.041(1)$ & $-0.004(1)$ & $0.037(1)$ & $0.001(1)$ \\
\hline$C(2 A)$ & $8 f$ & $0.3187(2)$ & $-0.2495(2)$ & $0.3123(1)$ & $0.055(2)$ & $0.058(2)$ & $0.030(1)$ & $0.009(1)$ & $0.019(1)$ & $-0.002(1)$ \\
\hline$C(3 A)$ & $8 f$ & $0.3501(2)$ & $-0.3290(3)$ & $0.2896(2)$ & $0.087(3)$ & $0.107(3)$ & $0.047(2)$ & $0.044(2)$ & $0.028(2)$ & $-0.005(2)$ \\
\hline$C(4 A)$ & $8 f$ & $0.4336(3)$ & $-0.3062(7)$ & $0.3180(3)$ & $0.078(3)$ & $0.285(9)$ & $0.055(3)$ & $0.092(5)$ & $0.013(2)$ & $-0.030(4)$ \\
\hline$C(5 A)$ & $8 f$ & $0.4347(3)$ & $-0.2129(8)$ & $0.2841(3)$ & $0.041(2)$ & $0.32(1)$ & $0.067(3)$ & $-0.003(4)$ & $0.028(2)$ & $-0.043(5)$ \\
\hline$C(6 A)$ & $8 f$ & $0.3990(2)$ & $-0.1368(4)$ & $0.3037(2)$ & $0.058(2)$ & $0.179(5)$ & $0.059(2)$ & $-0.046(3)$ & $0.034(2)$ & $-0.037(3)$ \\
\hline$N(7 A)$ & $8 f$ & $0.3193(1)$ & $-0.1610(2)$ & $0.2733(1)$ & $0.047(1)$ & $0.074(2)$ & $0.038(1)$ & $-0.018(1)$ & $0.025(1)$ & $-0.012(1)$ \\
\hline$C(8 A)$ & $8 f$ & $0.2849(2)$ & $-0.0809(2)$ & $0.2893(2)$ & $0.095(2)$ & $0.043(2)$ & $0.044(2)$ & $-0.019(1)$ & $0.042(2)$ & $-0.011(1)$ \\
\hline$C(9 A)$ & $8 f$ & $0.2071(2)$ & $-0.1024(2)$ & $0.2721(2)$ & $0.073(2)$ & $0.049(2)$ & $0.048(2)$ & $0.012(1)$ & $0.037(1)$ & $-0.003(1)$ \\
\hline$C(10 A)$ & $8 f$ & $0.1398(2)$ & $-0.1203(2)$ & $0.1811(2)$ & $0.064(2)$ & $0.070(2)$ & $0.051(2)$ & $0.016(2)$ & $0.030(2)$ & $0.005(2)$ \\
\hline$N(11 A)$ & $8 f$ & $0.1524(1)$ & $-0.2039(2)$ & $0.1487(1)$ & $0.046(1)$ & $0.061(1)$ & $0.037(1)$ & $-0.008(1)$ & $0.0216(9)$ & $-0.005(1)$ \\
\hline$C(12 A)$ & $8 f$ & $0.1718(2)$ & $-0.2868(2)$ & $0.1999(2)$ & $0.062(2)$ & $0.055(2)$ & $0.054(2)$ & $-0.016(1)$ & $0.036(1)$ & $-0.006(1)$ \\
\hline$C(13 A)$ & $8 f$ & $0.2136(2)$ & $-0.1883(2)$ & $0.3203(2)$ & $0.062(2)$ & $0.065(2)$ & $0.046(2)$ & $0.001(1)$ & $0.038(1)$ & $-0.000(1)$ \\
\hline
\end{tabular}


2. syn-11-Methyl-7,11-diazatricyclo $\left[7.3 .1 .0^{2,7}\right]$ tridecan-13-ol

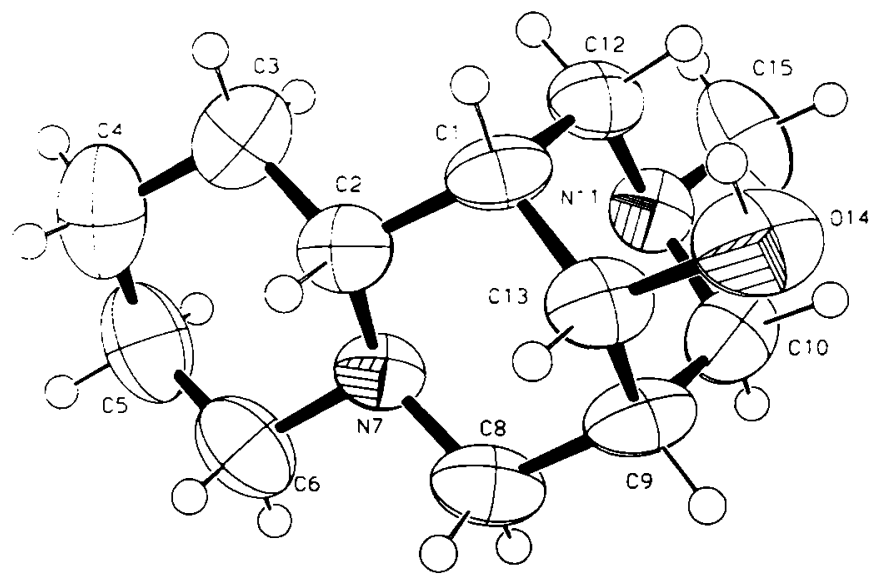

Table 4. Data collection and handling.

\begin{tabular}{ll}
\hline Crystal: & $\begin{array}{l}\text { colourless, thin laminar, } \\
\text { size } 0.025 \times 0.275 \times 1.0 \mathrm{~mm}\end{array}$ \\
Wavelength: & Mo $K_{\alpha}$ radiation $(0.71093 \AA)$ \\
$\mu:$ & $0.7 \mathrm{~cm}^{-1}$ \\
Diffractometer, scan mode: & Nonius CAD4, $\omega / 2 \theta$ \\
$2 \theta_{\text {max }}:$ & $45.96^{\circ}$ \\
$N(h k l)_{\text {measured, }} N(h k l)_{\text {unique: }}:$ & 3490,3304 \\
Criterion for $I_{\text {obs }}, N(h k l)_{\text {gt }}:$ & $I_{\text {obs }}>2 \sigma\left(l_{\text {obs }}\right), 1034$ \\
$N(\text { param })_{\text {refined: }}$ & 279 \\
Programs: & SHELXS-86 [13], SHELXL-93 [14], \\
& PLUTON93 [15], ORTEPII [16]
\end{tabular}

Table 5. Atomic coordinates and displacement parameters (in $\AA^{2}$ ).

\begin{tabular}{|c|c|c|c|c|c|}
\hline Atom & Site & $x$ & $y$ & $z$ & $U_{\text {iso }}$ \\
\hline$H(1)$ & $4 e$ & $0.5978(5)$ & $1.1732(5)$ & $0.3156(4)$ & 0.067 \\
\hline$H(2)$ & $4 e$ & $0.564 l(5)$ & $1.0605(6)$ & $0.4152(4)$ & 0.069 \\
\hline$H(31)$ & $4 e$ & $0.7499(5)$ & $1.0680(7)$ & $0.4175(4)$ & 0.097 \\
\hline$H(32)$ & $4 e$ & $0.7358(5)$ & $0.9524(7)$ & $0.3656(4)$ & 0.097 \\
\hline$H(41)$ & $4 e$ & $0.8145(7)$ & $0.8945(9)$ & $0.4934(4)$ & 0.131 \\
\hline$H(42)$ & $4 e$ & $0.7222(7)$ & $0.9615(9)$ & $0.5215(4)$ & 0.131 \\
\hline$H(51)$ & $4 e$ & $0.6900(7)$ & $0.7522(8)$ & $0.4288(4)$ & 0.116 \\
\hline$H(52)$ & $4 e$ & $0.6765(7)$ & $0.7617(8)$ & $0.5138(4)$ & 0.116 \\
\hline$H(61)$ & $4 e$ & $0.5027(6)$ & $0.7606(6)$ & $0.4193(4)$ & 0.094 \\
\hline$H(62)$ & 4e & $0.5194(6)$ & $0.8782(6)$ & $0.4697(4)$ & 0.094 \\
\hline H(81) & $4 e$ & $0.3759(5)$ & $0.9519(5)$ & $0.3645(4)$ & 0.081 \\
\hline$H(82)$ & $4 e$ & $0.3757(5)$ & $0.8420(5)$ & $0.3098(4)$ & 0.081 \\
\hline$H(9)$ & $4 e$ & $0.2984(5)$ & $1.0151(5)$ & $0.2389(4)$ & 0.075 \\
\hline$H(101)$ & $4 e$ & $0.3725(5)$ & $0.8798(6)$ & $0.1674(4)$ & 0.084 \\
\hline$H(102)$ & $4 e$ & $0.3838(5)$ & $1.0114(6)$ & $0.1400(4)$ & 0.084 \\
\hline$H(121)$ & $4 e$ & $0.5775(5)$ & $1.1093(5)$ & $0.1909(3)$ & 0.071 \\
\hline$H(122)$ & $4 e$ & $0.6697(5)$ & $1.0301(5)$ & $0.2465(3)$ & 0.071 \\
\hline $\mathrm{H}(13)$ & $4 e$ & $0.4138(5)$ & $1.1520(5)$ & $0.3249(4)$ & 0.071 \\
\hline$H(14)$ & $4 e$ & $0.437(8)$ & $1.27(1)$ & $0.232(7)$ & $0.24(6)$ \\
\hline$H(151)$ & $4 e$ & $0.6334(6)$ & $0.8974(6)$ & $0.1355(3)$ & 0.113 \\
\hline$H(152)$ & $4 e$ & $0.5306(6)$ & $0.9719(6)$ & $0.0879(3)$ & 0.113 \\
\hline$H(153)$ & $4 e$ & $0.5170(6)$ & $0.8356(6)$ & $0.1043(3)$ & 0.113 \\
\hline$H(1 A)$ & $4 e$ & $0.0994(5)$ & $0.5691(5)$ & $0.8231(3)$ & 0.060 \\
\hline$H(2 A)$ & $4 e$ & $0.0749(4)$ & $0.6855(5)$ & $0.9245(3)$ & 0.062 \\
\hline$H(3 A)$ & $4 e$ & $0.2571(5)$ & $0.6856(6)$ & $0.9146(4)$ & 0.082 \\
\hline H(3B) & $4 e$ & $0.2313(5)$ & $0.7997(6)$ & $0.8610(4)$ & 0.082 \\
\hline$H(4 A)$ & $4 e$ & $0.3212(6)$ & $0.8601(6)$ & $0.9874(4)$ & 0.104 \\
\hline H(4B) & $4 e$ & $0.2331(6)$ & $0.7936(6)$ & $1.0203(4)$ & 0.104 \\
\hline$H(5 A)$ & $4 e$ & $0.1844(6)$ & $0.9952(6)$ & $1.0077(4)$ & 0.093 \\
\hline$H(5 B)$ & $4 e$ & $0.1884(6)$ & $0.9963(6)$ & $0.9201(4)$ & 0.093 \\
\hline$H(6 A)$ & $4 e$ & $0.0305(5)$ & $0.8720(5)$ & $0.9762(3)$ & 0.073 \\
\hline H(6B) & $4 e$ & $0.0049(5)$ & $0.9849(5)$ & $0.9216(3)$ & 0.073 \\
\hline H(8A) & $4 e$ & $-0.1323(5)$ & $0.8889(5)$ & $0.8255(4)$ & 0.072 \\
\hline H(8B) & $4 e$ & $-0.1164(5)$ & $0.7807(5)$ & $0.8839(4)$ & 0.072 \\
\hline$H(9 A)$ & $4 e$ & $-0.2072(5)$ & $0.7112(5)$ & $0.7617(4)$ & 0.068 \\
\hline$H(10 A)$ & $4 e$ & $-0.1398(5)$ & $0.7161(5)$ & $0.6543(4)$ & 0.074 \\
\hline$H(10 B)$ & $4 e$ & $-0.1500(5)$ & $0.8481(5)$ & $0.6814(4)$ & 0.074 \\
\hline$H(12 A)$ & $4 e$ & $0.1542(5)$ & $0.7129(6)$ & $0.7447(3)$ & 0.074 \\
\hline$H(12 B)$ & $4 e$ & $0.0623(5)$ & $0.6268(6)$ & $0.6954(3)$ & 0.074 \\
\hline$H(13 A)$ & $4 e$ & $-0.0788(5)$ & $0.5810(5)$ & $0.8426(4)$ & 0.070 \\
\hline$H(14 A)$ & $4 e$ & $-0.049(7)$ & $0.46(1)$ & $0.735(6)$ & $0.23(5)$ \\
\hline$H(15 A)$ & $4 e$ & $0.0974(6)$ & $0.8339(6)$ & $0.6286(3)$ & 0.102 \\
\hline$H(15 B)$ & $4 e$ & $-0.0215(6)$ & $0.8913(6)$ & $0.6040(3)$ & 0.102 \\
\hline$H(15 C)$ & $4 e$ & $-0.0066(6)$ & $0.7546(6)$ & $0.5898(3)$ & 0.102 \\
\hline
\end{tabular}

Table 6. Atomic coordinates and displacement parameters (in $\AA^{2}$ ).

\begin{tabular}{|c|c|c|c|c|c|c|c|c|c|c|}
\hline Atom & Site & $x$ & $y$ & $z$ & $U_{11}$ & $U_{22}$ & $U_{33}$ & $U_{12}$ & $U_{13}$ & $U_{23}$ \\
\hline$C(1)$ & $4 e$ & $0.5591(5)$ & $1.0967(5)$ & $0.3014(4)$ & $0.055(4)$ & $0.031(4)$ & $0.084(5)$ & $-0.014(3)$ & $0.023(4)$ & $-0.008(4)$ \\
\hline$C(2)$ & $4 e$ & $0.5875(5)$ & $1.0178(6)$ & $0.3743(4)$ & $0.066(5)$ & $0.048(5)$ & $0.064(5)$ & $-0.004(4)$ & $0.025(4)$ & $-0.016(4)$ \\
\hline$C(3)$ & $4 e$ & $0.7101(5)$ & $0.9928(7)$ & $0.4057(4)$ & $0.071(5)$ & $0.082(5)$ & $0.085(5)$ & $0.001(4)$ & $0.012(4)$ & $-0.013(5)$ \\
\hline$C(4)$ & $4 e$ & $0.7364(7)$ & $0.9162(9)$ & $0.4787(4)$ & $0.117(7)$ & $0.127(9)$ & $0.069(6)$ & $0.028(7)$ & $0.004(6)$ & $0.000(6)$ \\
\hline$C(5)$ & $4 e$ & $0.6662(7)$ & $0.8043(8)$ & $0.4647(4)$ & $0.134(8)$ & $0.082(6)$ & $0.071(6)$ & $0.022(6)$ & $0.022(6)$ & $0.021(5)$ \\
\hline$C(6)$ & $4 e$ & $0.5454(6)$ & $0.8341(6)$ & $0.4311(4)$ & $0.110(7)$ & $0.059(5)$ & $0.074(5)$ & $0.009(5)$ & $0.041(5)$ & $0.009(4)$ \\
\hline$N(7)$ & $4 e$ & $0.5271(4)$ & $0.9054(4)$ & $0.3600(3)$ & $0.057(4)$ & $0.040(4)$ & $0.067(4)$ & $0.002(3)$ & $0.024(3)$ & $0.003(3)$ \\
\hline$C(8)$ & $4 e$ & $0.4085(5)$ & $0.9200(5)$ & $0.3252(4)$ & $0.073(5)$ & $0.040(4)$ & $0.101(6)$ & $0.000(4)$ & $0.044(5)$ & $0.006(4)$ \\
\hline $\mathrm{C}(9)$ & $4 e$ & $0.3790(5)$ & $1.0023(5)$ & $0.2539(4)$ & $0.049(4)$ & $0.030(4)$ & $0.110(6)$ & $-0.001(3)$ & $0.025(4)$ & $0.009(4)$ \\
\hline $\mathrm{C}(10)$ & $4 e$ & $0.4098(5)$ & $0.9556(6)$ & $0.1830(4)$ & $0.069(5)$ & $0.036(4)$ & $0.093(6)$ & $-0.002(4)$ & $0.003(4)$ & $0.009(4)$ \\
\hline $\mathrm{N}(11)$ & $4 e$ & $0.5286(4)$ & $0.9387(4)$ & $0.1976(3)$ & $0.064(4)$ & $0.033(3)$ & $0.063(4)$ & $0.004(3)$ & $0.016(3)$ & $-0.002(3)$ \\
\hline$C(12)$ & $4 e$ & $0.5908(5)$ & $1.0480(5)$ & $0.2311(3)$ & $0.063(4)$ & $0.039(4)$ & $0.077(5)$ & $-0.003(4)$ & $0.024(4)$ & $0.007(4)$ \\
\hline$C(13)$ & $4 e$ & $0.4348(5)$ & $1.1218(5)$ & $0.2792(4)$ & $0.061(5)$ & $0.031(4)$ & $0.088(5)$ & $0.003(3)$ & $0.023(4)$ & $0.006(4)$ \\
\hline$O(14)$ & $4 e$ & $0.3980(4)$ & $1.2029(5)$ & $0.2151(3)$ & $0.082(3)$ & $0.035(3)$ & $0.133(5)$ & $0.005(3)$ & $0.030(3)$ & $0.018(3)$ \\
\hline$C(15)$ & $4 e$ & $0.5546(6)$ & $0.9083(6)$ & $0.1252(3)$ & $0.153(7)$ & $0.065(5)$ & $0.069(5)$ & $0.014(5)$ & $0.040(5)$ & $0.009(4)$ \\
\hline$C(1 A)$ & $4 e$ & $0.0565(5)$ & $0.6431(5)$ & $0.8093(3)$ & $0.063(4)$ & $0.024(3)$ & $0.066(4)$ & $0.017(3)$ & $0.023(4)$ & $-0.004(3)$ \\
\hline$C(2 A)$ & $4 e$ & $0.0910(4)$ & $0.7264(5)$ & $0.8800(3)$ & $0.055(4)$ & $0.034(4)$ & $0.070(5)$ & $0.011(3)$ & $0.024(4)$ & $-0.002(3)$ \\
\hline$C(3 A)$ & $4 e$ & $0.2133(5)$ & $0.7585(6)$ & $0.9040(4)$ & $0.049(4)$ & $0.069(5)$ & $0.083(5)$ & $0.016(4)$ & $0.009(4)$ & $-0.001(4)$ \\
\hline$C(4 A)$ & $4 e$ & $0.2437(6)$ & $0.8369(6)$ & $0.9756(4)$ & $0.093(6)$ & $0.075(6)$ & $0.078(6)$ & $-0.008(5)$ & $0.001(5)$ & $-0.021(4)$ \\
\hline$C(5 A)$ & $4 e$ & $0.1704(6)$ & $0.9478(6)$ & $0.9602(4)$ & $0.111(6)$ & $0.053(5)$ & $0.061(5)$ & $-0.002(5)$ & $0.012(5)$ & $-0.013(4)$ \\
\hline
\end{tabular}


Table 6. Continued.

\begin{tabular}{|c|c|c|c|c|c|c|c|c|c|c|}
\hline Atom & Site & $x$ & $y$ & $z$ & $U_{11}$ & $U_{22}$ & $U_{33}$ & $U_{12}$ & $U_{13}$ & $U_{23}$ \\
\hline$C(6 A)$ & $4 e$ & $0.0500(5)$ & $0.9131(5)$ & $0.9338(3)$ & $0.085(5)$ & $0.041(4)$ & $0.062(4)$ & $0.011(4)$ & $0.031(4)$ & $-0.006(4)$ \\
\hline $\mathbf{N}(7 \mathbf{A})$ & $4 e$ & $0.0249(4)$ & $0.8358(4)$ & $0.8651(3)$ & $0.049(3)$ & $0.033(3)$ & $0.054(3)$ & $0.002(3)$ & $0.016(3)$ & $-0.008(3)$ \\
\hline$C(8 A)$ & $4 e$ & $-0.0936(5)$ & $0.8135(5)$ & $0.8402(4)$ & $0.054(4)$ & $0.046(4)$ & $0.086(5)$ & $0.010(3)$ & $0.030(4)$ & $-0.001(4)$ \\
\hline$C(9 A)$ & $4 e$ & $-0.1276(5)$ & $0.7277(5)$ & $0.7714(4)$ & $0.047(4)$ & $0.045(4)$ & $0.075(5)$ & $-0.001(3)$ & $0.011(4)$ & $-0.007(4)$ \\
\hline$C(10 A)$ & $4 e$ & $-0.1096(5)$ & $0.7736(5)$ & $0.6957(4)$ & $0.068(5)$ & $0.031(4)$ & $0.077(5)$ & $-0.001(3)$ & $0.006(4)$ & $-0.008(4)$ \\
\hline$N(11 A)$ & $4 e$ & $0.0072(4)$ & $0.7939(4)$ & $0.7011(3)$ & $0.067(4)$ & $0.043(3)$ & $0.045(3)$ & $-0.001(3)$ & $0.016(3)$ & $-0.001(3)$ \\
\hline$C(12 A)$ & $4 e$ & $0.0767(5)$ & $0.6904(6)$ & $0.7341(3)$ & $0.066(4)$ & $0.056(5)$ & $0.068(5)$ & $-0.002(4)$ & $0.026(4)$ & $-0.020(4)$ \\
\hline$C(13 A)$ & $4 e$ & $-0.0659(5)$ & $0.6109(5)$ & $0.7941(4)$ & $0.071(5)$ & $0.034(4)$ & $0.073(5)$ & $-0.002(4)$ & $0.026(4)$ & $-0.003(4)$ \\
\hline$O(14 A)$ & $4 e$ & $-0.1059(4)$ & $0.5259(4)$ & $0.7334(3)$ & $0.086(3)$ & $0.034(3)$ & $0.100(4)$ & $-0.005(3)$ & $0.014(3)$ & $-0.016(3)$ \\
\hline
\end{tabular}

Acknowledgments. The measurements were performed in FG Strukturforschung, FB Materialwissenschaft, Technische Universität Darmstadt, Petersenstr. 23, 64287 Darmstadt. The authors P. S. and P. N. Are indebted to the Hungarian National Research Foundation for the financial support (OTKAT021006).

\section{References}

1. Scheiber P.; Nemes, P.: Synthesis and stereochemistry of some new diazatricyclic compounds. Liebigs Ann. Chem. (1994) 1033-1036.

2. Brown, W. A. C.; Martin, J.; Sim, G. A.: Molecular conformations. I. The bicyclo[3.3.1]nonane system: $x$-ray analysis of 1 -( $p$-bromobenzenesulfonyloxymethyl)-5-methylbicyclo[3.3.1]nonanol. J. Chem. Soc. (1965) 1844.

3. Dobler, M.; Dunitz, J. D.: Structure of medium-size ring compounds. XI. Cycloactane-1,2-trans-dicarboxylic acid. Helv. Chim. Acta 47 (1967) 695.

4. Smith-Verdier, P.; Florescio, F.; S. Garcia-Blanco, S.: Structure of 3-benzyl-7-methyl-3,7-diazabicyclo[3.3.1]nonan-9-one, $\mathrm{C}_{15} \mathrm{H}_{20} \mathrm{~N}_{2} \mathrm{O}$. Acta Crystallogr. C39 (1983) 101-103.

5. Galvez, E.; Arias, M.S.; Bellanato, J.; Garcia-Ramos, J. V.; Florencio, F.; Smith-Verdier, P.; Garcia-Blanco, S.: Structural and conformational study of diazabicyclanones and diazabicyclanols. J. Mol. Struct. 127 (1985) 185-201.

6. Bailey III, B. R.; Berlin, K. D.; Holt, E. M.; Scherlag, B. J.; Lazzara, R.; Brachmann, J.; van der Helm, D.; Powell, D. R.; Pantaleo, N. S.; Ruenitz, P. C.: Synthesis, conformational-analysis, and antiarrhythmic properties of 7-benzyl-3-thia-7-azabicyclo[3.3.1]nonan-9-one, 7-benzyl-3-thia7-azabicyclo[3.3.1]nonane hydroperchlorate, and 7-benzyl-9-phenyl3-thia-7-azabicyclo[3.3.1]nonana-9-ol hydroperchlorate and derivates single crystal Xray-diffraction analysis and evidence for chair chair and chair boat conformers in the solid state. J. Med. Chem. 27 (1984) 758-767.

7. Thompson, M. D.; Smith, G. S.; Berlin, K. D.; Holt, E. M.; Scherlag, B. J. van der Helm, D.; Muchmore, S. W.; Fidelis, K. A.: Synthesis and antiarrhythmic properties of novel 3-selena-7- azabicyclo[3.3.1] Jnonanes and derivates. Single-crystal $x$-ray-diffraction analysis of 7-benzyl-3-selena-7-azabicyclo[3.3.1]nonan-9-one and 7-benzyl3-selena-7-azabicyclo[3.3.1]nonane hydroperchlorate. J. Med. Chem. 30 (1987) 780-788.
8. Jeyamaran, R.; Avila, S.: Chemistry of 3-azabicyclo[3.3.1]nonanes. Chem. Rev. 31 (1981) 149-174.

9. Balázs, B.; Nemes, P.; Scheiber, P.; Tóth, P. G.: Substituent and solvent dependent conformations of some saturated diazatricyclic compounds. J. Mol. Struct. 475 (1999) 153-160.

10. Barlow, R. B.; Johnson, O.: relations between structure and nicotine-like activity - $x$-ray crystal-structure analysis of $(-)$-cytisine and (-)-lobeline hydrochloride and a comparison with $(-)$-nicotine and other nicotine-like compounds. Br. J. Pharmacol. 98 (1989) 799-808.

11. Freer, A.; Robins, D. J.; Sheldrake, G. N.: Structures of (-)-Cytisine and $(-)$-methylcytisine - tricyclic quinolizidine alkaloids. Acta Crystallogr. C43 (1987) 1119-1122.

12. Mascagni, P.; Christodoulou, M.; Gibbons, W. A.; Asres, K.; Phillipson, J. D.; Niccolai, N.; Mangani, S.: Solution and crystal structure of cytisine, a quinolizidine alkaloid. J. Chem. Soc. Perkin Trans. II. (1987) 1 159-1 165.

13. Sheldrick, G. M.: SHELXS-86 Program for the solution of crystal structures. University of Göttingen, Germany 1986.

14. Sheldrick, G. M.: SHELXL-93 Program for crystal structure refinement. University of Göttingen, Germany 1993.

15. Spek, A. L.: PLUTON93. Program for the Display and Analysis of Crystal and Molecular Structures, Utrecht University, The Netherlands 1993.

16. Johnson, C. K.; Burnett, M. N.: ORTEP-III, Version 1.0.2, Farrugia, L. J. Windows 32-bit version 1.04, University of Glasgow, UK 1998. 